\title{
Laboreal
}

Volume $16 \mathrm{~N}^{\circ} 2$ | 2020

Programa de Pesquisa do Curso da Ação

\section{La ergotoxicología, de la palabra a los conceptos y metodologías}

A ergotoxicologia, da palavra aos conceitos e às metodologias

L'ergotoxicologie, du mot aux concepts et méthodologies

Ergotoxicology, from the word to concepts and methodologies

\section{Louis Galey y Alain Garrigou}

\section{(2) OpenEdition}

\section{Journals}

Edición electrónica

URL: http://journals.openedition.org/laboreal/16402

DOI: 10.4000/laboreal.16402

ISSN: 1646-5237

\section{Editor}

Universidade do Porto

\section{Referencia electrónica}

Louis Galey y Alain Garrigou, « La ergotoxicología, de la palabra a los conceptos y metodologías », Laboreal [En línea], Volume 16 N² | 2020, Publicado el 01 diciembre 220, consultado el 14 diciembre 2020. URL : http://journals.openedition.org/laboreal/16402; DOI : https://doi.org/10.4000/laboreal. 16402

Este documento fue generado automáticamente el 14 diciembre 2020.

Laboreal está licenciado com uma Licença Creative Commons - Atribuição-NãoComercial 4.0 Internacional. 


\title{
La ergotoxicología, de la palabra a los conceptos y metodologías
}

\author{
A ergotoxicologia, da palavra aos conceitos e às metodologias \\ L'ergotoxicologie, du mot aux concepts et méthodologies \\ Ergotoxicology, from the word to concepts and methodologies
}

Louis Galey y Alain Garrigou

\section{NOTA DEL EDITOR}

Traducción : María Noel Close - psicmariaclose@gmail.com

\section{Robert Villatte y la inclusión de la ergotoxicología en el proyecto de ergonomía de la actividad}

En Francia, cuando se publicó en 1985 el libro "Les risques du travail. Pour ne pas perdre sa vie à la gagner" (Los riesgos del trabajo, para no perder su vida ganándola) (Cassou et al, 1985), sólo habían transcurrido tres años desde las leyes Auroux que establecian la instauración de los Comités de Higiene, Seguridad y Condiciones de Trabajo, con nuevas prerrogativas en materia de condiciones de trabajo en la empresa. Estos cambios normativos reflejan las preocupaciones sociales sobre el trabajo y la salud. En ese momento, el libro, coordinado por médicos, médicos ocupacionales e inspectores de trabajo, es presentado por el Director General del Instituto Nacional de Salud e Investigación Médica de Francia (Inserm) y el epílogo está escrito por Alain Wisner. La publicación del libro fue un acontecimiento nacional que reunió 133 artículos y 106 autores, entre los que se encuentran ergónomos, médicos, médicos laborales, psiquiatras, estadistas, profesores-investigadores e ingenieros, en particular de instituciones tan diversas como el Instituto Nacional de Investigación y Seguridad (INRS) y las Cajas Regionales de Seguro de Enfermedad (CRAM), ahora CARSAT), el 
Instituto Nacional de Investigaciones Agrícolas (INRA), el Conservatorio Nacional de Artes y Oficios (Cnam), la Organización Profesional de Prevención de la Construcción y Obras Públicas (OPPBTP), el Inserm, el Centro Nacional de Investigaciones Científicas (CNRS) y el Ministerio de Trabajo.

2 En dicho libro, un texto de tres páginas titulado "Toxicología y ergonomía" de Robert Villatte (1985), cimentó las bases para un gran terreno de investigaciones e intervenciones sobre la temática del trabajo en presencia de productos químicos. Es la primera vez que el término "ergotoxicología" fue utilizado, en resonancia al texto precursor de Delvolvé (1984) publicado un año antes, en el que señalaba la importancia de considerar la particularidad de las actividades de trabajo en cuánto a modular los efectos de las exposiciones sobre la salud.

Robert Villatte, nació en 1946 y luego de una formación de técnico en física, trabajó en la industria farmacéutica y se convirtió en militante sindical en la Confédération française démocratique du travail (CFDT - Confederación Francesa Democrática del Trabajo - Federación de Química). En el contexto de sus mandatos como dirigente sindical, se interesó muy pronto por las consecuencias de la exposición de los trabajadores por turnos a productos tóxicos. Trabajando en colaboración con investigadores en el campo, se formó como ergónomo a tiempo completo en el Conservatorio Nacional de Artes y Oficios ( $\mathrm{Cnam}$ ) organizado por el equipo dirigido por Alain Wisner (1923-2004) dónde realizó sus primeras investigaciones de campo. Como señala Charles Gadbois (1937-2019) en la presentación de la entrevista realizada por la Comisión de Historia de la Sociedad de Ergonomía en Lengua Francesa (SELF), "su carrera estuvo marcada por la organización de la formación en ergonomía en el contexto sindical y la capitalización de los conocimientos mediante la publicación de artículos de revistas y libros" (Teiger et al., 2003). El texto "Toxicología y ergonomía" forma parte de este contexto. En ella, Villatte desarrolla el enfoque programático de la ergotoxicología a la luz de varias constataciones :

- "Considerar que existe un "hombre medio" con reacciones medias entra en contradicción con los conocimientos adquiridos sobre la dispersión de los parámetros fisiológicos en una población de individuos y su evolución a lo largo de la vida" (p.301) ;

- El impacto de los productos tóxicos en la salud varía en función de muchos factores (características del medio ambiente, variación del estado y la mezcla de los contaminantes en los talleres, naturaleza del trabajo, actividad desarrollada por el trabajador, etc.) ;

- Es necesario definir las prioridades de prevención en función de las actividades laborales.

4 Para Villatte, el simple cumplimiento de las normas no garantiza la ausencia de problemas de salud. La evolución de los conocimientos sobre la peligrosidad de los nuevos productos en el lugar de trabajo, su diversidad, las exposiciones múltiples, los protocolos y los procesos de elaboración de normas, además de la imposibilidad de tener en cuenta la singularidad de las actividades laborales en los estudios epidemiológicos o toxicológicos, subrayan la actualidad de este texto. 
5 Hoy en día, se han fortalecido las estrategias de prevención construidas en torno al uso de normas. Frente a los riesgos laborales que tienden a aumentar (DARES, 2019; Eurofound, 2017), uno de los pilares de la prevención de la exposición a productos químicos por inhalación se basa en el control de las atmósferas de trabajo mediante la medición, para su comparación con los valores límite de exposición laboral. Estos valores de referencia se elaboran a partir de los conocimientos científicos independientes de la Agencia Nacional de Seguridad Sanitaria (Anses) de Francia, que procura identificar los umbrales de exposición por encima de los cuales se observarían efectos indeseables. El ministerio encargado de la obra, por su parte, establece el proyecto de valor límite reglamentario. Por último, estos valores se someten a consulta social en el Consejo de Orientación de las Condiciones de Trabajo (COCT) bajo la égida del Ministerio de Trabajo (INRS \& ISSA, 2016).

6 En este contexto, las normas de toxicidad de los productos se elaboran siempre mediante soluciones intermedias basadas en los conocimientos científicos, y en cuestiones industriales y socioeconómicas en juego (Deveau et al., 2015). El ejemplo histórico del amianto (Henry, 2000), así como las diferencias entre países y las revisiones periódicas a la baja de los valores límite, nos recuerdan el posible desajuste entre los conocimientos científicos sobre los efectos para la salud y la reglamentación.

\section{La ergotoxicología hoy}

7 La ergotoxicología es definida como una práctica particular de la ergonomía (Garrigou, 2011) apunta a analizar las actividades de trabajo en los medios profesionales en dónde se puden producir exposición a productos químicos. En este marco la ergotoxicología desarrolla "modelos operativos (en el sentido de Wisner, 1972), herramientas y medios eficaces de prevención para gestionar y prevenir los riesgos para la salud de los trabajadores expuestos a los productos químicos" (Garrigou, 2011, p. 25).

8 En este sentido, es en efecto una forma de investigación e intervención con un objetivo operativo, profundamente arraigado en la ergonomía. En virtud de sus objetivos, la ergotoxicología moviliza conocimientos y modelos de la toxicología, la medicina laboral y la prevención. Hoy en día, la ergotoxicología no está reservada sólo a los ergónomos. También la movilizan especialistas en prevención, ergónomos y toxicólogos, ya sea en la propia empresa (internos) o en los servicios de salud ocupacional (MohammedBrahim et al., 2018), fomentando una práctica transprofesional.

9 Los fundamentos teóricos provienen del análisis de las actividades situadas (Leplat \& Cuny, 1977; Daniellou, 2005) y los enfoques culturalistas de la seguridad en las organizaciones (Morel et al., 2008) teniendo en cuenta las representaciones de los riesgos construidas en la acción individual y social (Duclos, 1987; Weill-Fassina, Rabardel \& Dubois, 1993), en una orientación de concepción de sistemas de trabajo (Jeffroy, 1987; Garrigou et al., 1995). El análisis de la actividad laboral, como punto de partida (Garrigou, 2011) para comprender las exposiciones, es una distinción esencial con la higiene industrial (Zartarian et al., 2004). De esta manera, la exposición se convierte en un objeto de frontera (Vinck, 2009) para animar los debates e intercambios, entre profesiones y disciplinas en el campo de la salud laboral.

10 Siguiendo el texto de Villatte, Sznelwar (1992) aplica la ergotoxicología a gran escala al tema de la exposición de los agricultores a los plaguicidas. Esta tesis, bajo la dirección 
de Alain Wisner, muestra el carácter operativo de la propuesta de Villatte. Los resultados destacan que la organización del trabajo y las estrategias de los operadores son aspectos fundamentales de la exposición, que los productores y operadores hacen compromisos y que es posible diferenciar la exposición según las actividades realizadas en cada etapa del trabajo. Este trabajo permite una comparación entre Francia y Brasil en la línea de la antropotecnología (Wisner, 1985), profundizando en la importancia de la dimensión psicológica de los trabajadores basada en la psicodinámica del trabajo.

11 La reanudación de las investigaciones en el ámbito de la agricultura (MohammedBrahim y otros, 1997) y la aplicación del enfoque ergotoxicológico en los trabajos de eliminación del amianto (Mohammed-Brahim et al., 1997), 1998) permitió reforzar la demostración de los límites de un modelo por pantalla (Mohammed-Brahim y Garrigou, 2009), ya sea de carácter normativo (mediante la aplicación de valores límite o la restricción del uso), basado en protecciones e instrucciones (en torno al uso de los EPI y los procedimientos que deben respetarse), o de carácter médico (en relación con las aptitudes e indicaciones médicas). Estos trabajos en la agricultura han abordado específicamente la falta de eficacia de los trajes protectores en situaciones reales de propagación en la vitivinicultura (Garrigou et al., 2011). En el mismo sentido, la demostración de que el proceso de homologación de los plaguicidas se basa en la prescripción del uso de EPI que es ineficaz en determinadas situaciones de trabajo refuerza el cuestionamiento global de las estrategias de prevención mediante el uso de pantallas (Garrigou et al., 2020). El paralelismo entre las "pantallas" descritas por Mohammed-Brahim \& Garrigou (2009) y las "normas" cuestionadas por Villatte (1985) es inevitable. Esta demostración en el campo de los plaguicidas confirma la fuerza de la propuesta inicial de Villatte. Se pone entonces en tela de juicio todo un paradigma funcionalista de prevención promulgado por los human factors (Reason, 1990, por ejemplo) [1].

12 En esta continuidad, se ha desarrollado una lógica de prevención construida a partir de la investigación sobre seguridad industrial (véase en particular Morel et al., 2008; Daniellou et al., 2011 ; Nascimento et al., 2014 ; Rocha et al., 2015), que permite probar las prescripciones de prevención. La implantación de este modelo se ha realizado principalmente en industrias químicas de riesgo (Garrigou et al., 2015; Galey et al., 2017; Mohammed-Brahim et al., 2018; Galey, 2019), articulando el análisis de situaciones de exposición reales (seguridad gestionada) para compararlas con las prescripciones de prevención (seguridad reglada), con el objetivo de desarrollar colectivamente los recursos de seguridad construidos [2]. Aunque este modelo tiene sus límites, a la hora de discernir lo reglado, lo gestionado y lo construido, por ejemplo, su operatividad como método de investigación de la intervención ha mostrado su interés en debatir las normas de seguridad en lo que respecta a la especificidad de las situaciones de trabajo, tal y como afirma Villatte, las exposiciones que experimentan los trabajadores y las representaciones de los riesgos asociados, en paralelo con el funcionamiento sistémico de las organizaciones.

13 Por lo tanto, la esencia de la ergotoxicología se centra en la resolución de enigmas (Teiger, 1993), lo que conduce a una comprensión de las exposiciones ocupacionales basada en el análisis de la actividad (Garrigou et al., 2004; Mohammed-Brahim \& Garrigou, 2009). Los actores de la empresa van a disponer de fragmentos de este enigma que necesitan ser ensamblados para entender la exposición. Esta comprensión requiere una movilización y una estrecha colaboración, similar en ciertos aspectos a una 
encuesta, con los múltiples actores internos o externos a la empresa, ya sean los operadores, la dirección directa o a distancia, los jefes de producción o el departamento de investigación y desarrollo, el médico laboral, etc. (Garrigou et al., 2004 ; MohammedBrahim \& Garrigou, 2009). Se trata de hacer coexistir y comprender diferentes "mundos" (Béguin, 2004, 2010), con sus propios sistemas de "coordenadas profesionales", en función de la forma de pensar, vivir y comprender las situaciones de trabajo (Judon, 2017). La actividad y sus huellas deben ser puestas en circulación en los medios profesionales para comprender sus causas a partir de los elementos subjetivos y objetivos aportados por los diferentes actores (Garrigou et al., 2004). Estas reflexiones sobre la intervención han nutrido un modelo de intervención ergotoxicológica (Mohammed-Brahim, 2015).

14 Con frecuencia se utilizan medidas para reforzar la comprensión general de la actividad. Estas medidas pueden ser fisiológicas (frecuencia cardíaca, respiración, angulaciones, etc.) o químicas (concentración del contaminante en tiempo real, muestreo por parche o filtro para análisis físico-químicos). Una observación sorprendente asociada al uso de la medida, cuando está vinculada a una descripción de la actividad, es la forma en que este instrumento ayuda a estimular los debates sobre las actividades laborales, las representaciones y las prácticas de prevención (Galey, 2019). La integración de la medida (utilizando la técnica video exposure monitoring, por ejemplo) es una estrategia de intervención que se convierte en un soporte para la transformación (Galey, Judon, Jolly et al., 2019).

Una particularidad de la ergotoxicología es que se basa en una diversidad de objetos intermedios (Vinck, 2009) para poner de manifiesto las actividades de exposición y la representación de los riesgos. Estos objetos intermedios toman la forma de mediciones, videos de la actividad, procedimientos de seguridad, etc. (Vinck, 2009). Su utilización en "espacios intermedios de prevención" (Judon, 2017 ; Judon et al., 2019) permite, por tanto, una comprensión diferente de las situaciones de exposición mediante un enfoque reflexivo, en el que las dimensiones íntimas del trabajo y la exposición, que contribuyen a establecer un vínculo entre la vida en el trabajo y fuera de él, se compartidos en presencia de los actores. En estas condiciones, se hace posible revelar el conocimiento detallado de los trabajadores sobre las situaciones de exposición a través de objetos intermedios. Los desarrollos metodológicos de las entrevistas de confrontación (Mollo \& Falzon, 2004) al asociar la medición en tiempo real con el vídeo son particularmente demostrativos en este punto (Judon et al., 2019). El lugar de las representaciones del riesgo se convierte en la clave para analizar las estrategias de los operadores y alimentar los enfoques de prevención (Judon, 2017 ; Judon et al., 2015). Los equipos de protección personal, por ejemplo, se están convirtiendo en un objeto de debate en los colectivos de trabajadores, lo que permite describir las contradicciones y compromisos de las estrategias de protección, como las formas de minimización (Duclos, 1987) y de negación del riesgo, o los límites de las prescripciones de prevención descendentes (Goutille et al., 2016). 


\section{Para concluir : Hacia una gestión de proyecto de diseño de prevención}

16 A partir de 2015, esta orientación progresiva hacia el diseño, se basa en la focalización en un enfoque centrado en el diagnóstico de las situaciones de exposición, sin alimentarse de la ergonomía de diseño para reforzar el potencial de transformación.

Los recientes trabajos en ergotoxicología se centran en los nuevos contaminantes cuyos riesgos para la salud se caracterizan por la incertidumbre (por ejemplo, las nanopartículas - Galey, Audignon, Brochard et al., 2020 - y el asfalto en las obras viales), al tiempo que se buscan posibilidades para desarrollar proyectos de diseño de prevención (Judon, 2017; Galey, 2019). Este contexto de incertidumbre lleva a la ausencia de "normas" de exposición específicas aceptadas para estos productos químicos. Un objetivo central es reducir la incertidumbre sobre las exposiciones reales en situaciones de trabajo haciéndolas visibles [3].

18 El desafío consiste entonces en caracterizar las situaciones de trabajo que implican actividades de exposición, con diferentes niveles de detalle y escalas de tiempo según las necesidades de las transformaciones o del diseño.

El abordaje se inspira en los trabajos pioneros de la ergonomía del diseño, en torno a la noción de la "situación de acción característica" (SAC) como unidad de análisis del trabajo para el diseño (Jeffroy, 1987 ; Garrigou, 1992 ; Daniellou, 1992 ; Garrigou et al., 1995 ; Daniellou, 2004; Duarte \& Lima, 2012; Barcellini et al., 2014), transpuesta en una unidad de análisis de los trabajos de exposición para concebir la prevención, utilizando "situaciones de exposición características" (SEC) (Galey, Audignon, Witschger et al., 2019; Galey, Audignon, Witschger et al., 2020). Estos resultados pueden integrarse en el diseño de una "fábrica del futuro" estableciendo procesos de fabricación aditiva de metales, o en una tesis pluriidisciplinaria entre el derecho y la ergonomía para cambiar la forma en que se concibe el material agrícola (Albert \& Garrigou, 2019). Esta ergotoxicología, que tiene sus raíces en las cuestiones sociotécnicas y reglamentarias contemporáneas, basada en las contribuciones de sus predecesores, permite en última instancia construir la prevención movilizando la medición y la actividad, reduciendo al mismo tiempo las incertidumbres.

Un cuestionamiento colectivo actual se refiere a las posibles evoluciones del término ergotoxicología, cuando se trata de producir conocimientos sobre las actividades de exposición para transformarlas, y no directamente conocimientos sobre la peligrosidad de los productos. El término "ergoexpología" podría ser apropiado, sin olvidar que el conocimiento de los posibles efectos tóxicos de los productos sigue siendo fundamental para definir las modalidades de la contaminación de los trabajadores y los posibles síntomas, así como los observables y las técnicas de medición que emergen del terreno. Al igual que la ergotoxicología, la ergoexpología estaría interesada en caracterizar las situaciones de exposición a los peligros que los trabajadores pueden encontrar en el curso de su trabajo, ya sean de origen químico, físico o biológico en particular. En este contexto, el objetivo de la ergoexpología sería comprender las actividades laborales relacionadas con estas exposiciones para prevenirlas y concebir sistemas de trabajo seguros.

21 Como en el caso de la ergotoxicología, nos parece que esta propuesta es, sin embargo, una posibilidad de subespecialización en ergonomía, así como un riesgo (pero también 
una oportunidad) para una comprensión más amplia del trabajo. Por ello, hay que recordar que la ergotoxicología (o ergoexpología) es ante todo un enfoque estructurado por los paradigmas fundadores de la ergonomía de la actividad, dirigido a los ergónomos y a todos los actores de la salud laboral que se enfrentan a los temas de la exposición profesional a los riesgos.

Una cosa es cierta : la "ergonomía vintage" [ $\left.{ }^{4}\right]$ aplicada a los nuevos desafíos del trabajo aún tiene un brillante futuro por delante.

\section{BIBLIOGRAFÍA}

Albert, M., \& Garrigou, A. (2019). Une approche critique de la conception et des usages du matériel de pulvérisation des pesticides en viticulture. Actes du 54ème Congrès de la Société d'Ergonomie de Langue Française (SELF). Comment contribuer à un autre monde ? Tours, France. Barcellini, F., Van Belleghem, L., \& Daniellou, F. (2014). Design projects as opportunities for the development of activities. In P. Falzon (Éd.), Constructive Ergonomics (pp. 187-204). CRC Press. https://doi.org/10.1201/b17456-16

Béguin, P. (2004). Mondes, monde commun et versions des mondes. Bulletin de psychologie, 57(469), $45-48$.

Béguin, P. (2010). Conduite de projet et fabrication collective du travail : Une approche développementale [Habilitation à diriger des recherches]. Laboratoire EVS UMR 5600, Université Victor Segalen Bordeaux 2, Bordeaux, France.

Cassou, B., Huez, D., Mousel, M.-L., Spitzer, C., \& Touranchet-Hébrard, A. (Dirs., 1985). Les risques du travail. Pour ne pas perdre sa vie à la gagner. Paris : La Découverte.

Daniellou, F. (1992). Le statut de la pratique et des connaissances dans l'intervention ergonomique de conception [Habilitation à diriger des recherches]. Laboratoire d'ergonomie des systèmes complexes, Université Victor Segalen-Bordeaux 2, Bordeaux, France.

Daniellou, F. (2004). 21. L'ergonomie dans la conduite de projets de conception de systèmes de travail. In P. Falzon, Ergonomie (1r éd., pp. 359-373). Paris : Presses Universitaires de France. https://doi.org/10.3917/puf.falzo.2004.01.0359

Daniellou, F. (2005). The French-speaking ergonomists' approach to work activity: Crossinfluences of field intervention and conceptual models. Theoretical Issues in Ergonomics Science, 6(5), 409-427. https://doi.org/10.1080/14639220500078252

Daniellou, F., Simard, M., \& Boissières, I. (2011). Human and organizational factors of safety: State of the art (Foundation for an Industrial Safety Culture). Toulouse : FonCSI.

DARES (2019). Comment ont évolué les expositions des salariés du secteur privé aux risques professionnels sur les vingt dernières années? Premiers résultats de l'enquête Sumer 2017 ( $\left.\mathrm{N}^{\circ} 41\right)$. Paris : Ministère du travail.

Delvolvé, N. (1984). Ergonomie et toxicologie. Le Travail Humain, 47(3), 227-235. 
Deveau, M., Chen, C.-P., Johanson, G., Krewski, D., Maier, A., Niven, K. J., Ripple, S., Schulte, P. A., Silk, J., Urbanus, J. H., Zalk, D. M., \& Niemeier, R. W. (2015). The Global Landscape of Occupational Exposure Limits-Implementation of Harmonization Principles to Guide Limit Selection. Journal of Occupational and Environmental Hygiene, 12(sup1), S127-S144. https://doi.org/ 10.1080/15459624.2015.1060327

Duarte, F., \& Lima, F. (2012). Anticiper l'activité par les configurations d'usage : Proposition méthodologique pour conduite de projet. Activités, 09(2). https://doi.org/10.4000/activites.314

Duclos, D. (1987). La construction sociale du risque : Le cas des ouvriers de la chimie face aux dangers industriels. Revue française de sociologie, 28(1), 17-42. https://doi.org/10.2307/3321444

Eurofound (2017). 6th European working conditions survey. Overview report [Publications Office of the European Union]. Bruxelles : Eurofound.

Galey, L. (2019). Comprendre les situations d'exposition aux nanoparticules par l'intégration de l'activité de travail à la mesure : Vers une construction de la prévention [Thèse de doctorat]. Ecole doctorale Sociétés, Politique, Santé Publique, Université de Bordeaux, Bordeaux, France.

Galey, L., Audignon, S., Brochard, P., Debia, M., Lacourt, A., Lambert, P., Bihan, O., Martinon, L., Pasquereau, P., Witschger, O., \& Garrigou, A. (2020). Towards an operational exposure assessment strategy to airborne nanoparticles by integrating work activity analysis and exposure measurement. Archives des Maladies Professionnelles et de l'Environnement, 81(3). https://doi.org/ 10.1016/j.admp.2020.03.831

Galey, L., Audignon, S., Witschger, O., Bau, S., Judon, N., Lacourt, A., \& Garrigou, A. (2020). What does ergonomics have to do with nanotechnologies? A case study. Applied Ergonomics, 87(103116). https://doi.org/10.1016/j.apergo.2020.103116

Galey, L., Audignon, S., Witschger, O., Lacourt, A., \& Garrigou, A. (2019). Toward a Better Assessment of Occupational Exposure to Nanoparticles Taking into Account Work Activities. In S. Bagnara, R. Tartaglia, S. Albolino, T. Alexander, \& Y. Fujita (Éds.), Proceedings of the 20th Congress of the International Ergonomics Association (IEA 2018) (pp. 465-478). Springer International Publishing. https://doi.org/10.1007/978-3-319-96089-0_50

Galey, L., Audignon-Durand, S., Witschger, O., Lacourt, A., \& Garrigou, A. (2017). Intégration de la sécurité dans l'innovation : Vers une meilleure caractérisation des expositions professionnelles aux nanoparticules ? Actes du 52e congrès de la SELF (pp. 169-173), Toulouse, France.

Galey, L., Judon, N., Jolly, C., Goutille, F., Morelot, S., Albert, M., Lhospital, O., Martin, P., NoelSuberville, C., Pasquereau, P., Aublet-Cuvelier, A., Mohammed-Brahim, B., \& Garrigou, A. (2019). Proposition méthodologique en ergotoxicologie pour révéler les expositions à des produits chimiques. Activités, 16(1). https://doi.org/DOI :10.4000/activites.4103

Garrigou, A. (1992). Les apports des confrontations d'orientation socio-cognitives au sein de processus de conception participatifs : Le rôle de l'ergonomie [Thèse de doctorat]. Laboratoire d'ergonomie, CNAM, Paris, France. http://www.theses.fr/1993CNAM0160

Garrigou, A. (2011). Le développement de l'ergotoxicologie : Une contribution de l'ergonomie à la santé au travail [Habilitation à Diriger des Recherches]. INSERM U1219, Université Victor Segalen Bordeaux 2, Bordeaux, France.

Garrigou, A., Baldi, I., Le Frious, P., Anselm, R., \& Vallier, M. (2011). Ergonomics contribution to chemical risks prevention: An ergotoxicological investigation of the effectiveness of coverall against plant pest risk in viticulture. Applied Ergonomics, 42(2), 321-330. https://doi.org/10.1016/ j.apergo.2010.08.001 
Garrigou, A., Daniellou, F., Carballeda, G., \& Ruaud, S. (1995). Activity analysis in participatory design and analysis of participatory design activity. International Journal of Industrial Ergonomics, 15(5), 311-327. https://doi.org/10.1016/0169-8141(94)00079-I

Garrigou, A., Laurent, C., Berthet, A., Colosio, C., Jas, N., Daubas-Letourneux, V., Jackson Filho, J.M., Jouzel, J.-N., Samuel, O., Baldi, I., Lebailly, P., Galey, L., Goutille, F., \& Judon, N. (2020). Critical review of the role of PPE in the prevention of risks related to agricultural pesticide use. Safety Science, 123(104527). https://doi.org/10.1016/j.ssci.2019.104527

Garrigou, A., Peeters, S., Jackson, M., Sagory, P., \& Carballeda, G. (2004). 30. Apports de l'ergonomie à la prévention des risques professionnels. In P. Falzon, Ergonomie (1r éd., pp. 497-514). Paris : Presses Universitaires de France. http://www.cairn.info/ ergonomie--9782130514046-page-497.htm

Garrigou, A., Théry, L., Chassaing, K., Effantin, E., Mercieca, P., Dimerman, S., Vanderghote, M., Negroni, P., Gauthier, P., Goutille, F., Galey, L., Rambaud, C., Laporte, E., Merlin, X., Vergneaux, L., \& Baratta, R. (2015). Une approche pluridisciplinaire du processus de construction sociale de la prévention du risque CMR. Actes du 50ème congrès de la SELF (pp. 476-489), Paris, France.

Goutille, F., Galey, L., Rambaud, C., Pasquereau, P., Jackson, M., \& Garrigou, A. (2016). Prescrição e utilização de equipamentos de proteção individual (EPI) em atividades com exposição a produtos químicos cancerígenos, mutagênicos e reprotóxicos (CMR) : Pesquisa-ação pluridisciplinar em uma fábrica francesa de decoração para móveis. Laboreal, 12(1). https://doi.org/10.15667/ laborealxii0116fg

Henry, E. (2000). Un scandale improbable. Amiante : D’une maladie professionnelle à une “crise de santé publique» [Thèse de doctorat]. Université de Technologie de Compiègne.

INRS, \& ISSA. (2016). ED 6254 Valeurs limites d'exposition pour la prévention des risques chimiques (pp. 82). Retrieved from http://www.inrs.fr/media.html?refINRS=ED\%206254

Jeffroy, F. (1987). Maîtrise de l'exploitation d'un système micro-informatique par des utilisateurs noninformaticiens : Analyse ergonomique et processus cognitif [Thèse de doctorat]. Paris 13, Paris, France.

Jouzel, J.-N. (2019). Pesticides. Comment ignorer ce que l'on sait. Paris : SciencesPo Les Presses.

Judon, N. (2017). Rendre possible un espace intermédiaire de dialogue pour co-construire de nouvelles solutions de prévention dans un contexte d'incertitude. Cas des travaux de revêtements routiers [Thèse de doctorat]. Ecole doctorale Sociétés, Politique, Santé Publique, Université de Bordeaux, Bordeaux, France.

Judon, N., Galey, L., Saint Dizier de Almeida, V., \& Garrigou, A. (2019). Contributions of participatory ergonomics to the involvement of workers in chemical risk prevention projects. Work, 64(3), 651-660. https://doi.org/10.3233/WOR-193001

Judon, N., Hella, F., Pasquereau, P., \& Garrigou, A. (2015). Vers une prévention intégrée du risque chimique lié à l'exposition cutanée au bitume des travailleurs de la route. Élaboration d'une méthodologie dans le cadre de l'ergotoxicologie. Perspectives interdisciplinaires sur le travail et la santé, 17(2). https://doi.org/10.4000/pistes.4586

Leplat, J., \& Cuny, X. (1977). Introduction à la psychologie du travail (1ère édition). Paris : PUF.

Mohammed-Brahim, B. (2015). L'ergotoxicologie ou comment aborder le risque chimique du point de vue de l'activité du travail. Actes de la troisième Conférence internationale sur Les applications de l'ergonomie dans les pays en voie de développement, 6. Alger, Algérie. Retrieved from http:// prevention-ergonomics.com/ar/Paper\%2014\%20Brahim\%20MOHAMMEDBRAHIM\%20\%20Ergo\%202015.pdf 
Mohammed-Brahim, B., Daniellou, F., Brochard, P. (1997). “ Non pas que nous ne raisonnons pas, mais nous raisonnons autrement »: A propos d'un regard de l'ergonome sur la conduite raisonnée du traitement pesticide en viticulture. Actes du 32e congrès de la SELF (pp. 107-115), Lyon, France.

Mohammed-Brahim, B., Daniellou, F., Garrigou, A. (1998). Amiante: Chantier de retrait secteur 2. Guide pour le médecin du travail. pp. 125. Bordeaux : AHI 33.

Mohammed-Brahim, B., \& Garrigou, A. (2009). Une approche critique du modèle dominant de prévention du risque chimique. Activités, 06(1). https://doi.org/10.4000/activites.2086

Mohammed-Brahim, B., Garrigou, A., Treiber, A., Clement, N., Cuesta, C., Balent, I., Brun, G., \& Josse, P. (2018). Ergotoxicologie : État de l'art. Des pistes pour l'intervention à partir de retours d'expériences diversifiés. Actes du 53e congrès de la SELF (pp. 369-389), Bordeaux, France.

Mollo, V., \& Falzon, P. (2004). Auto- and allo-confrontation as tools for reflective activities. Applied Ergonomics, 35(6), 531-540. https://doi.org/10.1016/j.apergo.2004.06.003

Morel, G., Amalberti, R., \& Chauvin, C. (2008). Articulating the Differences Between Safety and Resilience: The Decision-Making Process of Professional Sea-Fishing Skippers. Human Factors: The Journal of the Human Factors and Ergonomics Society, 50(1), 1-16. https://doi.org/ $10.1518 / 001872008 X 250683$

Nascimento, A., Cuvelier, L., Mollo, V., Dicioccio, A., \& Falzon, P. (2014). Constructing safety. In Constructive Ergonomics (pp. 95-110). CRC Press.

Reason, J. (1990). Human Error. New York: Cambridge University Press.

Rocha, R., Mollo, V., \& Daniellou, F. (2015). Work debate spaces: A tool for developing a participatory safety management. Applied Ergonomics, 46, 107-114. https://doi.org/10.1016/ j.apergo.2014.07.012

Sznelwar, L. I. (1992). Analyse ergonomique de l'exposition de travailleurs agricoles aux pesticides. Essai ergotoxicologique [Thèse de doctorat]. Laboratoire d'ergonomie, CNAM, Paris, France.

Teiger, C. (1993). L'approche ergonomique : Du travail humain à l'activité des hommes et des femmes au travail. Education Permanente, 3(116), 27.

Teiger, C., Laville, A., \& Gadbois, C. (2003). Robert Villatte. Entretien réalisé par Catherine Teiger et Antoine Laville (p. 9) [Commission Histoire de la Société d'Ergonomie de Langue Française].

Villatte, R. (1985). Toxicologie et ergonomie. In B. Cassou, D. Huez, M.-L. Mousel, C. Spitzer \& A. Touranchet-Hébrard (Dirs), Les risques du travail. Pour ne pas perdre sa vie à la gagner (pp. 301-303). Paris: La Découverte.

Vinck, D. (2009). From Intermediary Object towards Boundary-Object. Revue d'anthropologie des connaissances, 3(1), 51-72.

Weill-Fassina, A., Rabardel, P., \& Dubois, D. (Coord., 1993). Représentations pour l'action. Toulouse : Octarès.

Wisner, A. (1972). Diagnosis in Ergonomics or the Choice of Operating Models in Field Research. Ergonomics, 15(6), 601-620. https://doi.org/10.1080/00140137208924462

Wisner, A. (1985). Quand voyagent les usines. Essai d'anthropologie. Paris : Syros

Zartarian, V., Bahadori, T., \& McKone, T. (2004). Adoption of an official ISEA glossary. Journal of Exposure Science and Environmental Epidemiology. https://doi.org/10.1038/sj.jea.7500411 


\section{NOTAS}

1. Podemos incluso preguntarnos acerca del modo en que estas normas y pantallas constituyen un modo de poner en relieve algunos saberes sobre los riesgos, y no otros (Jouzel, 2019).

2. El término "seguridad" es utilizado con respecto al resultado global de las acciones de prevención y uso de las protecciones en el medio profesional. La utilización del término es influenciada por la noción de seguridad del trabajo o aún de las investigaciones de seguridad en el mundo industrial y hospitalario. Este término "seguridad" designa un trabajo "seguro" sin problemas de salud, desde una perspectiva durable y del desarrollo.

3. A partir de mediciones y vídeos de situaciones de trabajo, sus análisis y selecciones en función de lo observado y las hipótesis subyacentes, sus usos y circulación con los actores de la empresa.

4. Alain Garrigou denomina así a la ergotoxicología, debido a las técnicas movilizadas con apariencias tradicionales, como la medición o el análisis de la actividad.

\section{AUTORES}

\section{LOUIS GALEY}

Équipe TE2O, LAPPS (EA4386), Université Paris Nanterre, 200 avenue de la République, 92001 Nanterre Cedex, France Équipe ergonomie, CRTD (EA4132), Cnam, 41 rue Gay Lussac, 75005 Paris, France louis.galey@parisnanterre.fr

\section{ALAIN GARRIGOU}

EPICENE, U1219 INSERM Bordeaux Population Health Research Center, Université de Bordeaux, 146 rue Leo Saignat, 33076 Bordeaux Cedex, France alain.garrigou@u-bordeaux.fr 